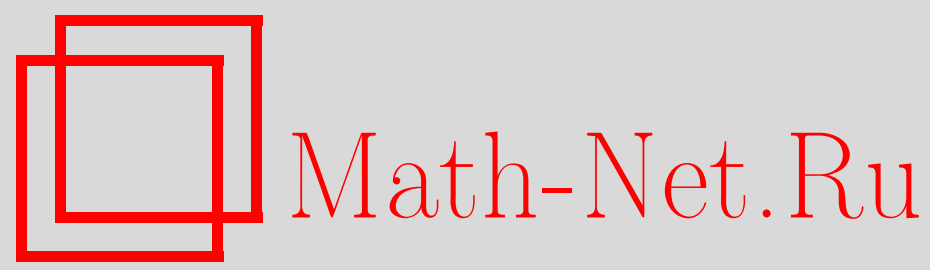

В. Н. Салий, Квазибулевы степени элементарных абелевых р-групп, Матем. заметки, 1999, том 66, выпуск 2, 264-274

DOI: https://doi.org/10.4213/mzm1164

Использование Общероссийского математического портала Math-Net.Ru подразумевает, что вы прочитали и согласны с пользовательским соглашением http://www.mathnet.ru/rus/agreement

Параметры загрузки:

IP : 3.81 .55 .215

26 апреля 2023 г., 11:56:32

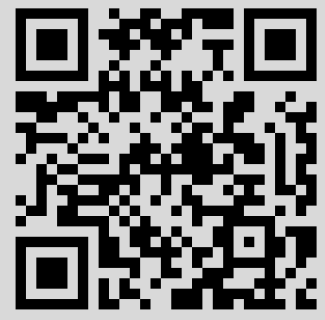




\title{
КВАЗИБУЛЕВЫ СТЕПЕНИ \\ ЭЛЕМЕНТАРНЫХ АБЕЛЕВЫХ $p$-ГРУПП
}

\section{В.Н. Салий}

\begin{abstract}
Основной результат работы (теорема 3) - характеризация группоидов с единицей, допускающих вложение в квазибулевы степени элементарных абелевых $p$-групп при $p>2$.

Библиография: 11 названий.
\end{abstract}

Понятие булевой степени алгебры играет важную роль в общей теории алгебраических систем (см. [1], [2]). Близким его обобщением является конструкция квазибулевой степени алгебры. Сохраняя многие свойства исходного понятия, она имеет и ряд существенных отличий. Одно из них касается многообразий алгебр. Известно, что для любого многообразия $V$ все булевы степени $V$-алгебр также принадлежат $V$. Для квазибулевых степеней подобной закономерности нет. Так, если все многообразия унарных алгебр замкнуты относительно квазибулевых степеней [3], то среди полугрупповых многообразий этим свойством обладает только многообразие полугрупп с нулевьм умножением [4]. Последнее обстоятельство создает новые классификационные возможности для группоидов (алгебр с одной бинарной операцией), позволяя связать с каждьп многообразием $V$ полугруп классы групоидов, так или иначе определяемые квазибулевыми степенями $V$-алгебр. В этом смысле естественно начать с минимальных многообразий полугруп. Кроме упомянутого многообразия полугрупп с нулевым умножением, выделяемого тождеством $x y=z t$, в этот список входят [5, c. 162]: многообразие левосингулярных полугрупш (полугрупп левых нулей) $x y=x$; многообразие правосингулярных полугрупп (полугрупп правых нулей) $x y=y$; многообразие полурешеток $x^{2}=x$, $x y=y x$; многообразие элементарных абелевых $p$-групп для каждого простого $p$ с определяющими тождествами $x y=y x, x^{p} y=y$. В работе [6] был описан класс (квазимногообразие) группоидов, вложимьх в квазибулевы степени левосингулярных полугрупп (из двойственных соображений получается описание соответствующего класса для правосингулярных полугрупп); в сообщении [7] анонсировано решение аналогичной задачи о характеризации подгруппоидов в квазибулевьх степенях полурешеток (развернутое изложение в печати). Результаты настоящей заметки в основном завершают исследование минимальных многообразий полугрупп в обсуждаемом аспекте: найдены необходимые и достаточные условия, при выполнении которых группоид с единищей допускает вложение в подходящую квазибулеву степень некоторой элементарной абелевой $p$-группы при заданном $p>2$. Случай $p=2$ не укладьвается в общую схему и требует дополнительного рассмотрения. 
Пусть $L$ - полная решетка. Под ортогональной системой в ней понимается всякое подмножество $\left\{l_{i} \mid i \in I\right\}$ такое, что $l_{i} \wedge l_{j}=0$ при $i \neq j$ и $\bigvee_{i \in I} l_{i}=1$. Ортогональная система $\left\{l_{i} \mid i \in I\right\}$ по определению независима, если $\bigvee_{j \in J} l_{j} \wedge \bigvee_{k \in K} l_{k}=0$ для любого разбиения $I=J \cup K, J \cap K=\varnothing$. Полная решетка с дополнениями называется квазибулевой, если в ней все ортогональные системы независимы. Это свойство имеет место, например, в каждой полной булевой решетке. Квазибулевой решеткой является и пятиугольник $N_{5}$ - пятиэлементная немодулярная решетка. Полная решетка с дополнениями тогда и только тогда будет квазибулевой, когда она допускает $\wedge$-гомоморфизм на полную булеву решетку, который сохраняет точные верхние грани ортогональных систем и, кроме того, взаимно однозначен в 0 и 1 (см. [8] и [5, с. 271]). Такой $\wedge$-гомоморфизм назьвается каноническим.

Пусть $(G, \cdot)$ - группа, т.е. полугруппа с единицей, где каждый элемент имеет обратный, и $L-$ квазибулева решетка. Через $G[L]$ обозначим совокупность всех отображений $\nu: G \rightarrow L$ таких, что

1) $\nu(f) \wedge \nu(g)=0$ при $f \neq g$

2) $\bigvee_{f \in G} \nu(f)=1$.

В множестве $G[L]$ вводится умножение по формуле

$$
(\mu \nu)(f)=\bigvee_{f=g h}(\mu(g) \wedge \nu(h))
$$

для любых $\mu, \nu \in G[L], f \in G$. Группоид $(G[L], \cdot)$ назьвается $L$-степенью группы $G$. Когда $L$ пробегает класс всех квазибулевых решеток, получаем квазибулевы степени группы $G$. Среди них находятся и все ее булевы степени, если ограничиться только полными булевыми решетками $L$. Булева степень любой группы сама является групой.

A ссоциацией называется группоид с единицей, в котором каждьй элемент имеет обратный и умножение ассоциативно в единице, т.е. в произведениях равных единице скобки можно расставлять произвольно. Каждая группа, очевидно, является ассоциацией. Минимальньй пример ассоциации, отличной от групшы, дает трехэлементньй группоид $A=\{e, a, b\}$, где $e$ - единица и $a a=a b=b a=b b=e$. Группоид с единицей тогда и только тогда будет ассоциацией, когда он допускает гомоморфизм на группу, взаимно однозначньй в единице. Этот гомоморфизм также назьвается каноническим. Ядром канонического гомоморфизма ассоциации является конгруэнция $\theta$, отождествляющая два элемента тогда и только тогда, когда эти элементы совместно обратимы, т.е. у них одни и те же обратные (см. [8]).

Пусть $\mathscr{K}$ - некоторый класс алгебр, $\mathscr{B}$ и $\mathscr{C}$ - его подклассы. Мальиевским $\mathscr{K}$-произведением классов $\mathscr{B}$ и $\mathscr{C}$ назьвается класс $\mathscr{A}=\mathscr{B}$ о $\mathscr{K} \mathscr{C}$, состоящий из всех $\mathscr{K}$-алгебр $A$, на которых существует конгруэнция $\theta$ такая, что каждый ее класс, являющийся $\mathscr{K}$-алгеброй, принадлежит $\mathscr{B}$, а факторалгебра $A / \theta$ принадлежит $\mathscr{C}$ (см. [9]).

Теорема 1. Класс $\mathscr{A}$ всех ассоциаций совпадает с мальцевским произведением $\mathscr{E}$ о $\mathscr{G}$, әде Г - класс всех группоидов с единицей, $\mathscr{E}-$ его тривиальный подкласс, $\mathscr{G}$ - класс всеx әpynn.

ДокАЗАТЕЛЬСтво. Если $\varphi: A \rightarrow G-$ канонический гомоморфизм ассоциации $A$ на группу $G$, то конгруэнция $\theta=\operatorname{Ker} \varphi$ имеет единственный класс, замкнутый относительно умножения в $A$. Это одноэлементное множество $\{e\}$, состоящее из единицы. Значит, $\mathscr{A} \subseteq \mathscr{E} \circ_{\Gamma} \mathscr{G}$. 
$\mathrm{C}$ другой стороны, пусть группоид с единищей $A$ допускает конгруэнцию $\varepsilon$ такую, что $A / \varepsilon$ - группа, а все $\varepsilon$-классы, являющиеся подгруппоидами в $A$, одноэлементны. Так как единственным $\varepsilon$-классом, замкнутьм относительно умножения в $A$, является $\varepsilon(e)$, то $\varepsilon(e)=\{e\}$. Таким образом, естественное отображение nat $\varepsilon: A \rightarrow A / \varepsilon$ будет гомоморфизмом группоида $A$ на группу $A / \varepsilon$, взаимно однозначным в единице. Следовательно, $A-$ ассоциация и $\mathscr{E}$ ог $\mathscr{G} \subseteq \mathscr{A}$.

Следующее предложение выясняет роль ассоциаций в рамках решаемой задачи.

ТЕОРема 2. Все квазибулевы степени групп являются ассоциациями.

ДокАЗАТЕЛЬСтво. Пусть $G$ - группа и $L-$ квазибулева решетка. Если $e-$ единица группы $G$, то единицей группоида $G[L]$ является $\nu_{e}: G \rightarrow L$ такое, что $\nu_{e}(f)=1$ при $f=e$ и $\nu_{e}(f)=0$ в противном случае. Пусть $\varphi_{0}: L \rightarrow L^{*}, l \mapsto l^{*},-$ канонический $\wedge$-гомоморфизм квазибулевой решетки $L$ на полную булеву решетку $L^{*}$. Из $[10$, теорема 3] следует, что отображение $\varphi: G[L] \rightarrow G\left[L^{*}\right], \nu \mapsto \nu^{*}$, такое, что $\nu^{*}(f)=(\nu(f))^{*}$ для любых $\nu \in G[L], f \in G$, является гомоморфизмом группоида $G[L]$ на групш $G\left[L^{*}\right]$. Поскольку $\varphi_{0}$ взаимно однозначно в 0 и 1 , единственным $\varphi$-прообразом единицы $\nu_{e}$ группы $G\left[L^{*}\right]$ будет единица $\nu_{e}$ группоида $G[L]$. Следовательно, $G[L]-$ ассоциация.

Абелева группа назьвается әлементарной, если порядки ее неединичных элементов не делятся на квадрат. В элементарной абелевой $p$-группе порядок любого неединичного элемента равен $p$ (простое число). Каждая такая группа разлагается в прямое произведение циклических групп $Z_{p}$ порядка $p$ (см. [11, теорема 8.5]).

Следующая теорема описьвает группоиды с единицей, вложимые в квазибулевы степени элементарных абелевых $p$-групп при $p>2$.

ТЕорема 3. Группоид с единицей вкладывается в подходящую квазибулеву степень $A$ некоторой әлементарной абелевой $p$-группы при $p>2$ тогда и только тогда, когда

1) А является коммутативной ассоциацией;

2) подгруппоид $\langle\alpha\rangle$, порожденный любым неединичным әлементом $\alpha \in A$, изоморфен $Z_{p}$

3) $(\alpha \beta)^{n}=\alpha^{n} \beta^{n}, 1 \leqslant n<p$, для любых $\alpha, \beta \in A$;

4) отношение $\alpha \leqslant \beta \Longleftrightarrow(\alpha, \beta) \in \theta \& \alpha \beta=\alpha \alpha$ является стабильным порядком на $A$ (здесь $\theta$ - отношение совместной обратимости әлементов uз $A)$;

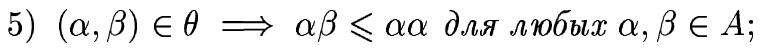

6) $(\alpha \alpha) \beta \leqslant \alpha(\alpha \beta)$ для любых $\alpha, \beta \in A$.

ДокАЗАтЕльство. Необходимость. Пусть $G$ - элементарная абелева $p$-группа, $p>2$, и $L$ - квазибулева решетка. Покажем, что в квазибулевой степени $G[L]$ выполняются все условия 1)-6).

1) В теореме 2 доказано, что $G[L]$ - ассоциация. Коммутативность следует из определения умножения в $G[L]$ (формула (1)).

Для дальнейшего потребуется

ЛЕмма 1. Если $\nu \in G[L]$, mо $\nu^{[n]}(f)=\nu(\sqrt[n]{f})$ для любого $f \neq e$, где $\nu^{[n]}-$ произведение $n$ сомножстелей $\nu$ с произвольной расстановкой скобок. 
ДОКАЗАТЕЛЬСТВо. При $n=2$ имеем

$$
(\nu \nu)(f)=\bigvee_{f=g h}(\nu(g) \wedge \nu(h))=\left\{\begin{array}{ll}
0 & \text { при } g \neq h, \\
\nu(g) & \text { при } g=h,
\end{array}=\nu(\sqrt{f}),\right.
$$

т.е. $\nu^{2}(f)=\nu(\sqrt{f})$.

Пусть утверждение справедливо для всех $m<n<p$. Так как $\nu^{[n]}=\nu^{[k]} \nu^{[l]}$, где $k+l=n$, то

$$
\nu^{[n]}(f)=\left(\nu^{[k]} \nu^{[l]}\right)(f)=\bigvee_{f=g h}\left(\nu^{[k]}(g) \wedge \nu^{[l]}(h)\right)=\bigvee_{f=g h}(\nu(\sqrt[k]{g}) \wedge \nu(\sqrt[l]{h}))=\nu(\sqrt[k]{g}),
$$

где $f=g \sqrt[k]{g}$, откуда $\nu^{[n]}(f)=\nu(\sqrt[n]{f})$. Лемма 1 доказана.

2) Из леммы 1 следует, что умножение степеней любого элемента $\nu \in G[L]$ ассоциативно. Пусть $\langle\nu\rangle$ - наименьшая подассоциация ассоциации $G[L]$, содержашая элемент $\nu \neq \nu_{e}$. Так как булева степень $G\left[L^{*}\right]$, где $L^{*}$ - канонический $\wedge$-гомоморфный образ квазибулевой решетки $L$, является $p$-группой, все неединичные циклические подгруппы в $G\left[L^{*}\right]$ изоморфны $Z_{p}$. Тогда $\langle\nu\rangle / \theta \cong Z_{p}$. Согласно лемме 1 в каждом $\theta$-классе находится не более одного элемента из $\langle\nu\rangle$. Значит, $\langle\nu\rangle \cong Z_{p}$.

3) Используя лемму 1 , для любых $\mu, \nu \in G[L]$ получаем

$$
\begin{aligned}
(\mu \nu)^{n}(f) & =(\mu \nu)(\sqrt[n]{f})=\bigvee_{\sqrt[n]{f=g h}}(\mu(g) \wedge \nu(h))=\bigvee_{\sqrt[n]{f}=g h}\left(\mu^{n}\left(g^{n}\right) \wedge \nu^{n}\left(h^{n}\right)\right) \\
& =\bigvee_{f=g^{n} h^{n}}\left(\mu^{n}\left(g^{n}\right) \wedge \nu^{n}\left(h^{n}\right)\right)=\left(\mu^{n} \nu^{n}\right)(f),
\end{aligned}
$$

т.е. $(\mu \nu)^{n}=\mu^{n} \nu^{n}$.

Элементы $\mu, \nu \in G[L]$ назьваются совместимы.ми, если $\mu(f) \wedge \nu(g)=0$ при $f \neq g$. Оказывается, что отношение совместимости в $G[L]$ совпадает с отношением совместной обратимости $\theta$. Именно, имеет место

Лемма 2. Если $\mu, \nu \in G[L], \quad$ то $(\mu, \nu) \in \theta$ тогда и только тогда, когда $\mu(f) \wedge \nu(g)=0$ npu $f \neq g$.

ДокАЗАТЕльСтво. Пусть $(\mu, \nu) \in \theta$. Одним из обратных для $\mu$ будет элемент $\mu^{-1}$, определяемый равенством $\mu^{-1}(f)=\mu\left(f^{-1}\right)$ для любого $f \in G$. Он должен быть обратным и для $\nu$, т.е. $\mu^{-1} \nu=\nu_{e}$. Пусть $f, g$-различные элементы группы $G$. Тогда

$$
\mu(f) \wedge \nu(g)=\mu^{-1}\left(f^{-1}\right) \wedge \nu(g) \leqslant \bigvee_{u v \neq e}\left(\mu^{-1}(u) \wedge \nu(v)\right)=\left(\mu^{-1} \nu\right)(u v)=0,
$$

так как $u v \neq e$. Итак, $\mu(f) \wedge \nu(g)=0$ при $f \neq g$.

Обратно, пусть $\mu(f) \wedge \nu(g)=0$ при $f \neq g$ и $\rho$ - обратный для $\mu$ элемент в $G[L]$, т.е. $\mu \rho=\nu_{e}$. Тогда при каноническом гомоморфизме ассоциации $G[L]$ на группу $G\left[L^{*}\right]$ (см. доказательство теоремы 2) получаем

$$
(\nu \rho)^{*}=\nu^{*} \rho^{*}=\mu^{*} \rho^{*}=(\mu \rho)^{*}=\nu_{e}^{*}=\nu_{e},
$$

откуда $\nu \rho=\nu_{e}$, т.е. $\rho$ - обратньй для $\nu$ элемент в $G[L]$. Лемма 2 доказана.

Следуюшая лемма показывает, что естественньй порядок " $\leqslant$ " в $G[L]$ (поточечное сравнение: $\mu \leqslant \nu \Longleftrightarrow \mu(f) \leqslant \nu(f)$ для всех $f \in G$ ) может быть выражен в терминах умножения. 
ЛЕмма 3. Если $\mu, \nu \in G[L]$, mo $\mu \leqslant \nu \Longleftrightarrow(\mu, \nu) \in \theta \& \mu \nu=\mu \mu$.

ДокАЗАТЕЛЬСТво. Если $\mu \leqslant \nu$, то, конечно, $\mu$ и $\nu$ совместимы, откуда по лемме 2 $(\mu, \nu) \in \theta$. Используя лемму 1 , имеем

$$
(\mu \nu)(f)=\bigvee_{f=g h}(\mu(g) \wedge \nu(h))=\mu(\sqrt{f}) \wedge \nu(\sqrt{f})=\mu(\sqrt{f})=(\mu \mu)(f)
$$

т.е. $\mu \nu=\mu \mu$.

С другой стороны, если $(\mu, \nu) \in \theta$ и $\mu \nu=\mu \mu$, то $\mu(f) \wedge \nu(f)=(\mu \nu)\left(f^{2}\right)=\mu(f)$ для любого $f \in G$, откуда $\mu \leqslant \nu$. Лемма 3 доказана.

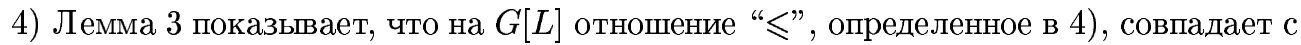
естественным порядком “ $\leqslant$ ", который стабилен относительно умножения (1).

5) Пусть $(\mu, \nu) \in \theta$. Используя леммы 1 и 2 , получаем

$$
(\mu \nu)(f)=\bigvee_{f=g h}(\mu(g) \wedge \nu(h))=\mu(\sqrt{f}) \wedge \nu(\sqrt{f}) \leqslant \mu(\sqrt{f})=(\mu \mu)(f)
$$

т.е. $\mu \nu \leqslant \mu \mu$.

6) Непосредственно вычисляя, получаем

$$
\begin{aligned}
(\mu(\mu \nu))(f) & =\bigvee_{f=g h}(\mu(g) \wedge(\mu \nu)(h))=\bigvee_{g}\left(\mu(g) \wedge(\mu \nu)\left(f g^{-1}\right)\right) \\
& =\bigvee_{g}\left(\mu(g) \wedge \bigvee_{f g^{-1}=x y}(\mu(x) \wedge \nu(y))\right)=\bigvee_{g}\left(\mu(g) \wedge \bigvee_{x}\left(\mu(x) \wedge \nu\left(f g^{-1} x^{-1}\right)\right)\right) \\
& \geqslant \bigvee_{g}\left(\mu(g) \wedge\left(\mu(g) \wedge \nu\left(f g^{-1} g^{-1}\right)\right)\right)=\bigvee_{g}\left(\mu(g) \wedge \nu\left(f g^{-1} g^{-1}\right)\right) \\
& =\bigvee_{g}\left((\mu \mu)(g g) \wedge \nu\left(f g^{-1} g^{-1}\right)\right)=\bigvee_{f=g h}((\mu \mu)(g) \wedge \nu(h))=((\mu \mu) \nu)(f),
\end{aligned}
$$

т.е. $(\mu \mu) \nu \leqslant \mu(\mu \nu)$.

Таким образом, в квазибулевых степенях элементарных абелевых $p$-груп, $p>2$, выполняются все условия 1)-6). Следовательно, они вьполняются и в группоидах с единицей, вложимых в эти квазибулевы степени.

Достаточность. Пусть группоид $A$ с единицей $e$ удовлетворяет условиям 1)-6). Так как вьполняется 1 ), отношение $\theta$ совместной обратимости является конгруэнцией на $A$ и факторгрупп $G=A / \theta$ - абелевой. Как следствие 2$),$ в $G$ истинно тождество $x^{p}=e$. Таким образом, $G=A / \theta-$ элементарная абелева $p$-группа.

Построим вложение групшы $G$ в булеву степень $Z_{p}\left[L^{*}\right]$, где $L^{*}=P(G)$ - множество всех подмножеств множества $G$.

Группа $G$ является прямым произведением циклических груп $G_{i}, i \in I$, каждая из которых изоморфна $Z_{p}$. Элементы группы $G$ можно считать функциями $f: I \rightarrow Z_{p}$, для которых множества $\operatorname{supp} f:=\{i \in I \mid f(i) \neq e\}$ конечны. Если $\operatorname{supp} f \subseteq\left\{i_{1}, i_{2}, \ldots, i_{n}\right\}$, то элемент $f \in G$ можно записать в виде $f=g_{i_{1}}^{k_{1}} g_{i_{2}}^{k_{2}} \cdots g_{i_{n}}^{k_{n}}$, где $g_{i_{m}}$ - фиксированный неединичньй элемент группы $G_{i_{m}} \cong Z_{p}, 1 \leqslant m \leqslant n$. Понятно, что если $i_{m} \notin \operatorname{supp} f$, то $k_{i_{m}}=0$. 
Сопоставим элементу $f \in G$ отображение $\chi_{f}: G \rightarrow Z_{p}$ следующим образом. Если $g \in G$ и $z$ - фиксированный неединичный элемент в $Z_{p}$, то при $\left\{i_{1}, i_{2}, \ldots, i_{n}\right\} \supseteq \operatorname{supp} f \cup$ $\operatorname{supp} g$ полагаем

$$
\chi_{f}(g)=\chi_{f}\left(g_{i_{1}}^{l_{1}} g_{i_{2}}^{l_{2}} \cdots g_{i_{n}}^{l_{n}}\right):=z^{k_{1} l_{1}+k_{2} l_{2}+\cdots+k_{n} l_{n}} .
$$

Если $\left\{i_{1}, i_{2}, \ldots, i_{n}\right\} \supseteq \operatorname{supp} f \cup \operatorname{supp} g \cup \operatorname{supp} h$, то

$$
\begin{aligned}
\chi_{f}(g h) & =\chi_{f}\left(g_{i_{1}}^{l_{1}+m_{1}} g_{i_{2}}^{l_{2}+m_{2}} \cdots g_{i_{n}}^{k_{n}+m_{n}}\right)=z^{k_{1}\left(l_{1}+m_{1}\right)+k_{2}\left(l_{2}+m_{2}\right)+\cdots+k_{n}\left(l_{n}+m_{n}\right)} \\
& =z^{k_{1} l_{1}+k_{2} l_{2}+\cdots+k_{n} l_{n}} z^{k_{1} m_{1}+k_{2} m_{2}+\cdots+k_{n} m_{n}}=\chi_{f}(g) \chi_{f}(h) .
\end{aligned}
$$

Значит, $\chi_{f} \in \operatorname{Hom}\left(G, Z_{p}\right)$.

Соответствие $f \mapsto \chi_{f}$ взаимно однозначно. В самом деле, если $f \neq g$, то $g_{i}^{k_{i}} \neq g_{i}^{l_{i}}$ для некоторого $i \in \operatorname{supp} f \cup \operatorname{supp} g$. Но тогда $\chi_{f}\left(g_{i}\right)=z^{k_{i}} \neq z^{l_{i}}=\chi_{g}\left(g_{i}\right)$, т.е. $\chi_{f} \neq \chi_{g}$.

Соответствие $f \mapsto \chi_{f}$, как нетрудно проверить, сохраняет умножение, т.е. $\chi_{f g}(h)=$ $\chi_{f}(h) \chi_{g}(h)$ для любых $f, g, h \in G$ и, следовательно, является вложением группы $G$ в ее группу характеров $\operatorname{Hom}\left(G, Z_{p}\right)$.

Теперь каждому элементу $f \in G$ сопоставим отображение $\nu_{f}=\chi_{f}^{-1}: Z_{p} \rightarrow L^{*}$, где $\chi_{f}: G \rightarrow Z_{p}$ - характер, соответствующий $f$, а $L^{*}=P(G)$. Легко понять, что $\chi_{f}^{-1}(u) \cap \chi_{f}^{-1}(v)=\varnothing$ при $u \neq v$ и $\bigcup_{z \in Z_{p}} \chi_{f}^{-1}(z)=G$, так что $\nu_{f} \in Z_{p}\left[L^{*}\right]$.

Далее,

$$
\begin{aligned}
\left(\nu_{f} \nu_{g}\right)(z) & =\bigcup_{z=u v}\left(\nu_{f}(u) \cap \nu_{g}(v)\right)=\bigcup_{z=u v}\left(\chi_{f}^{-1}(u) \cap \chi_{g}^{-1}(v)\right) \\
& =\bigcup_{z=u v}\left(\left\{h \in G \mid \chi_{f}(h)=u\right\} \cap\left\{h \in G \mid \chi_{g}(h)=v\right\}\right) \\
& =\bigcup_{z=u v}\left\{h \in G \mid \chi_{f}(h)=u \& \chi_{g}(h)=v\right\} \\
& =\left\{h \in G \mid \chi_{f}(h) \chi_{g}(h)=z\right\}=\left\{h \in G \mid \chi_{f g}(h)=z\right\}=\chi_{f g}^{-1}(z)=\nu_{f g}(z)
\end{aligned}
$$

и, значит, $\nu_{f g}=\nu_{f} \nu_{g}$.

Поскольку при $f \neq g$ характеры $\chi_{f}$ и $\chi_{g}$ различны, то в этом случае и $\nu_{f} \neq \nu_{g}$.

Итак, группа $G=A / \theta$ вложена в булеву степень $Z_{p}\left[L^{*}\right]$, где $L^{*}=P(G)$. Трансформацией этого представления и будет получено вложение группоида $A$ в группод $Z_{p}[L]$, где $L$ - квазибулева решетка, к построению которой мы теперь переходим.

Пусть $S:=\{\langle\alpha\rangle \mid \alpha \in A\}$ - множество всех однопорожденных подгруппоидов группоида $A$. Согласно 2) каждьй подгруппоид $\langle\alpha\rangle$ изоморфен групе $Z_{p}$. Очевидно, что если $\langle\alpha\rangle \neq\langle\beta\rangle$, то $\langle\alpha\rangle \cap\langle\beta\rangle=\{e\}$.

Используя порядок на $A$, определенньй в 4$)$, введем на множестве $S$ одноименное отношение " $\leqslant$ ", полагая

$$
\langle\alpha\rangle \leqslant\langle\beta\rangle \Longleftrightarrow\left(\exists \alpha_{0} \in\langle\alpha\rangle, \beta_{0} \in\langle\beta\rangle\right)\left(\alpha_{0} \leqslant \beta_{0}\right)
$$

Лемма 4. Если $\langle\alpha\rangle \leqslant\langle\beta\rangle$, то для любых $\gamma \in\langle\alpha\rangle, \delta \in\langle\beta\rangle$ из $(\gamma, \delta) \in \theta$ следует $\gamma \leqslant \delta$ 
ДокАЗАтЕльство. В самом деле, если $\langle\alpha\rangle \leqslant\langle\beta\rangle$, то существуют $\alpha_{0} \in\langle\alpha\rangle, \beta_{0} \in\langle\beta\rangle$ такие, что $\left(\alpha_{0}, \beta_{0}\right) \in \theta$ и $\alpha_{0} \beta_{0}=\alpha_{0} \alpha_{0}$. Пусть $\gamma \in\langle\alpha\rangle, \delta \in\langle\beta\rangle$ и $(\gamma, \delta) \in \theta$. Тогда $\gamma=\alpha_{0}^{k}$, $\delta=\beta_{0}^{k}$ для некоторого $k<p$. Ссылаясь на аксиому 3$)$, получаем

$$
\gamma \delta=\alpha_{0}^{k} \beta_{0}^{k}=\left(\alpha_{0} \beta_{0}\right)^{k}=\left(\alpha_{0} \alpha_{0}\right)^{k}=\alpha_{0}^{k} \alpha_{0}^{k}=\gamma \gamma
$$

и, значит, $\gamma \leqslant \delta$.

ЛЕмма 5. Отношение "К” является порядком на $S$.

ДоказАтельство. Рефлексивность очевидна. Далее, если $\langle\alpha\rangle \leqslant\langle\beta\rangle$ и $\langle\beta\rangle \leqslant\langle\gamma\rangle$, то, считая $(\alpha, \beta) \in \theta$ и $(\beta, \gamma) \in \theta$, получаем $\alpha \leqslant \beta$ и $\beta \leqslant \gamma$, откуда $\alpha \leqslant \gamma$ и, значит, $\langle\alpha\rangle \leqslant\langle\gamma\rangle,-$ отношение " $\leqslant$ " транзитивно на $S$. Для доказательства антисимметричности допустим, что $\langle\alpha\rangle \leqslant\langle\beta\rangle$ и $\langle\beta\rangle \leqslant\langle\alpha\rangle$, причем $(\alpha, \beta) \in \theta$. Тогда $\alpha \leqslant \beta$ и $\beta \leqslant \alpha$, т.е. $\alpha \beta=\alpha \alpha$ и $\beta \alpha=\beta \beta$. В силу коммутативности умножения $\alpha \alpha=\beta \beta$. Таким образом, $\alpha \alpha \in\langle\alpha\rangle \cap\langle\beta\rangle$. Так как $\alpha \alpha \neq e$ (по условию $p>2$ ), то $\langle\alpha\rangle=\langle\beta\rangle$.

ЛЕмма 6. Если $\langle\alpha\rangle \leqslant\langle\gamma\rangle u\langle\beta\rangle \leqslant\langle\delta\rangle$, mo $\langle\alpha \beta\rangle \leqslant\langle\gamma \delta\rangle$.

ДокАЗАТЕльство. Действительно, если $\alpha \leqslant \gamma$ и $\beta \leqslant \delta$, то в силу 4 ) (стабильность порядка " $\leqslant$ " на $A$ ) вьполнено $\alpha \beta \leqslant \gamma \delta$, откуда $\langle\alpha \beta\rangle \leqslant\langle\gamma \delta\rangle$.

Положим $S(\alpha):=\{\langle\beta\rangle \mid \beta \in \theta(\alpha)\}$, т.е. $S(\alpha)$ - это множество подгруппоидов в $A$, порожденных элементами $\theta$-класса, содержащего $\alpha$.

ЛЕмма 7. Упорядоченное подмножество $(S(\alpha), \leqslant)$ упорядоченного множества $(S, \leqslant)$ при любом $\alpha$ является полурешеткой, где $\inf (\langle\gamma\rangle,\langle\delta\rangle)=\langle\gamma \delta\rangle$.

ДокАЗАтЕЛЬСтво. Пусть $\langle\beta\rangle \in S(\alpha)$. Будем считать, что $(\alpha, \beta) \in \theta$. Согласно 5) $\alpha \beta \leqslant \alpha \alpha$, откуда $\langle\alpha \beta\rangle \leqslant\langle\alpha \alpha\rangle=\langle\alpha\rangle$. Аналогично, $\langle\alpha \beta\rangle \leqslant\langle\beta\rangle$. Если $\langle\gamma\rangle \leqslant\langle\alpha\rangle$ и $\langle\gamma\rangle \leqslant\langle\beta\rangle$, то, считая $(\alpha, \gamma) \in \theta$, получаем $\gamma \leqslant \alpha$ и $\gamma \leqslant \beta$, откуда вследствие 4) $\gamma \gamma \leqslant \alpha \beta$ и, значит, $\langle\gamma\rangle=\langle\gamma \gamma\rangle \leqslant\langle\alpha \beta\rangle$. Таким образом, циклическая группа $\langle\alpha \beta\rangle$ является наибольшей нижней гранью для циклических групп $\langle\alpha\rangle$ и $\langle\beta\rangle$ в $(S(\alpha), \leqslant)$.

Упорядоченные множества $(S, \leqslant)$ и $(S(\alpha), \leqslant), \alpha \in A$, играют определяющую роль в построении квазибулевой решетки $L$.

Под идеалами упорядоченного множества понимаются его минорантно насьщенные подмножества. Наименьший идеал, содержаший подмножество $X$, обозначается чеpeз $I(X)$.

Пусть $L(\alpha):=\operatorname{Id}(S(\alpha))$ - решетка идеалов полурешетки $(S(\alpha), \leqslant)$. В булевой решетке $L^{*}=P(G)$ каждьй элемент вида $\chi_{\theta(\alpha)}^{-1}(z), z \in Z_{p}$, заменим решеткой $L(\alpha)$, обозначая ее в этой ситуации через $L(\alpha, z)$. Каждьй элемент $l$, лежащий в $L^{*}$ ниже элемента вида $\chi_{\theta(\alpha)}^{-1}(z)$ и отличньй от $\varnothing$, заменим решеткой $\operatorname{Id}(S \times S)$ идеалов прямого квадрата $(S \times S, \leqslant)$ упорядоченного множества $(S, \leqslant)$, обозначая ее в этой ситуации через $L[l]$ (заметим, что в $L^{*}$ различные элементы вида $\chi_{\theta(\alpha)}^{-1}(z)$ несравнимы).

Теперь определим порядок в множестве $L$, полученном из $L^{*}$ в результате всех указанных замен. Сохраняя все порядковые связи, имеющиеся в решетках вида $L(\alpha, z)$ и $L(l)$, зададим дополнительно следуюшие соотношения:

а) наименьший элемент 0 решетки $L^{*}$ (т.е. $\varnothing$ ) остается наименьшим элементом в $L$;

б) если $\chi_{\theta(\alpha)}^{-1}(z)<l$ в $L^{*}$, то в $L$ каждьй элемент подрешетки $L(\alpha, z)$ считается меньше $l$; 
в) если $0<l_{1}<l_{2}<\chi_{\theta(\alpha)}^{-1}(z)$ в $L^{*}$ для некоторых $\alpha$ и $z$, то каждьй элемент подрешетки $L\left(l_{1}\right)$ считается меньше одноименного элемента подрешетки $L\left(l_{2}\right)$;

г) если $0<l<\chi_{\theta(\alpha)}^{-1}(z)$ в $L^{*}$ для некоторых $\alpha$ и $z$, то считается, что наибольшим минорантом элемента $I \in L(\alpha, z)$ в подрешетке $L(l)$ является идеал

$$
J:=I(\{(\langle\gamma\rangle,\langle\delta\rangle) \in S \times S \mid\langle\gamma\rangle \in I, \text { или }\langle\delta\rangle \in I, \text { или }\langle\gamma \delta\rangle \in I\}) .
$$

Транзитивное замыкание построенного отношения, состоящего из объединения порядков в решетках вида $L(\alpha, z)$ и $L(l)$ и дополнительных связей, введенных в а)-г), упорядочивает множество $L$.

Покажем, что $L$ с введенньм порядком является решеткой. Необходимо рассмотреть два неочевидных случая.

Пусть $I_{1}, I_{2} \in L(\alpha, z)$. В $L(\alpha, z)$ наибольшей нижней гранью для $\left\{I_{1}, I_{2}\right\}$ будет $I_{1} \cap I_{2}$. Но у этих идеалов есть общие нижние грани и в каждой подрешетке вида $L(l)$, где $l<$ $\chi_{\theta(\alpha)}^{-1}(z)$. Пусть $\chi_{\theta(\alpha)}^{-1}(z)$ является покрытием для $l$ в $L^{*}$. Наибольшей нижней гранью для $\left\{I_{1}, I_{2}\right\}$ в $L(l)$ будет пересечение идеалов

$$
J_{i}=I\left(\left\{(\langle\gamma\rangle,\langle\delta\rangle) \in S \times S \mid\langle\gamma\rangle \in I_{i}, \text { или }\langle\delta\rangle \in I_{i}, \text { или }\langle\gamma \delta\rangle \in I_{i}\right\}\right), \quad i=1,2 .
$$

Пусть $(\langle\gamma\rangle,\langle\delta\rangle) \in J_{1} \cap J_{2}$. Из возможных девяти случаев типичными являются следующие три:

a) $\langle\gamma\rangle \in I_{1},\langle\gamma\rangle \in I_{2}$

б) $\langle\gamma\rangle \in I_{1},\langle\delta\rangle \in I_{2}$

в) $\langle\gamma\rangle \in I_{1},\langle\gamma \delta\rangle \in I_{2}$.

В случае а) $\langle\gamma\rangle \in I_{1} \cap I_{2}$. В случае б) в полурешетке $S(\alpha)$ имеем $\langle\gamma \delta\rangle=\inf (\langle\gamma\rangle,\langle\delta\rangle)$, откуда $\langle\gamma \delta\rangle \leqslant\langle\gamma\rangle \in I_{1}$ и $\langle\gamma \delta\rangle \leqslant\langle\delta\rangle \in I_{2}$ и, значит, $\langle\gamma \delta\rangle \in I_{1} \cap I_{2}$. В случае в) так как $\langle\gamma\rangle \in S(\alpha)$ и $\langle\gamma \delta\rangle \in S(\alpha)$, то $\theta(\gamma)$ и $\theta(\gamma \delta)$ будут элементами циклической группы $\cup S(\alpha) / \theta$. Поскольку $\theta(\gamma \delta)=\theta(\gamma) \cdot \theta(\delta)$ в $A / \theta$, то $\langle\delta\rangle \in S(\alpha)$. Теперь имеем $\langle\gamma \delta\rangle=$ $\inf (\langle\gamma\rangle,\langle\delta\rangle) \leqslant\langle\gamma\rangle \in I_{1}$ и, значит, $\langle\gamma \delta\rangle \in I_{1}$, так что $\langle\gamma \delta\rangle \in I_{1} \cap I_{2}$.

Таким образом, $J_{1} \cap J_{2}<I_{1} \cap I_{2}$ в $L$ и, следовательно, $\inf _{L}\left(I_{1}, I_{2}\right)=I_{1} \cap I_{2}$.

Пусть $J_{1}, J_{2} \in L(l)$, причем $l$ имеет в $L^{*}$ одним из своих покрытий элемент $\chi_{\theta\left(\alpha_{0}\right)}^{-1}\left(z_{0}\right)$. В $L[l]$ наименьшей верхней гранью для $\left\{J_{1}, J_{2}\right\}$ будет $J_{1} \cup J_{2}$. Но у этих идеалов есть общие верхние грани и вне $L[l]$. Наименьшей из них является

$$
\bigcup_{\alpha \in A} \bigcup_{i=1}^{2}\left(I\left(\operatorname{pr}_{1} J_{i} \cap S(\alpha)\right) \cup I\left(\operatorname{pr}_{2} J_{i} \cap S(\alpha)\right) \cup I\left(\left\{\langle\gamma \delta\rangle \mid\langle\gamma\rangle,\langle\delta\rangle \in J_{i}\right\} \cap S(\alpha)\right)\right),
$$

где $\operatorname{pr}_{1} J$ - множество всех первых элементов пар, входящих в отношение $J \subseteq S \times S$, а $\mathrm{pr}_{2} J$ - множество всех вторых элементов этих пар. Если финальное объединение (по элементам $\alpha \in A$ ) содержит не менее двух различных членов, оно даст элемент решетки $L$ больший, чем наибольший элемент подрешетки $L\left(\alpha_{0}, z_{0}\right)$ и, значит, больший, чем $J_{1} \cup J_{2}$. Пусть $\operatorname{pr}_{1} J_{i} \subseteq S\left(\alpha_{0}\right), \operatorname{pr}_{2} J_{i} \subseteq S\left(\alpha_{0}\right)$ и $\langle\gamma \delta\rangle \in S\left(\alpha_{0}\right)$ для каждой пары $(\langle\gamma\rangle,\langle\delta\rangle) \in J_{i}, i=1,2$. Тогда наименьшей верхней гранью для $\left\{J_{1}, J_{2}\right\}$ в $L\left(\alpha_{0}, z_{0}\right)$ будет объединение $\operatorname{pr}_{1}\left(J_{1} \cup J_{2}\right) \cup \operatorname{pr}_{2}\left(J_{1} \cup J_{2}\right) \cup I\left(\left\{\langle\gamma \delta\rangle \mid(\langle\gamma\rangle,\langle\delta\rangle) \in J_{1} \cup J_{2}\right\}\right)$, которое мажорирует $J_{1} \cup J_{2}$. Таким образом, $\sup _{L}\left(J_{1}, J_{2}\right)=J_{1} \cup J_{2}$. 
Мы получили, что $L$ - решетка. Очевидно, что она будет полной. Если в $L$ отождествить элементы каждой подрешетки вида $L(\alpha, z)$ и отождествить элементы каждой подрешетки вида $L(l)$, получится канонический $\wedge$-гомоморфизм решетки $L$ на булеву решетку $L^{*}=P(G)$. Следовательно, $L-$ квазибулева решетка.

Покажем, что группоид $A$ вкладьвается в квазибулеву степень $Z_{p}[L]$.

Для большей наглядности элементы подрешеток вида $L(\alpha, z)$ будем записьвать в виде пар $\left(I, \chi_{\theta(\alpha)}^{-1}(z)\right)$ и вместо $I(\langle\alpha\rangle)$ писать $I(\alpha)$.

Определим отображение $\varphi: A \rightarrow Z_{p}[L], \alpha \mapsto \nu_{\alpha}$, полагая $\nu_{e}(z)=1$ при $z=e$ и $\nu_{e}(z)=0$ в противном случае, и при $\alpha \neq e$, полагая $\nu_{\alpha}(z)=\left(I(\alpha), \chi_{\theta(\alpha)}^{-1}(z)\right), z \in Z_{p}$.

Поскольку $\chi_{\theta(\alpha)}^{-1}(u) \cap \chi_{\theta(\alpha)}^{-1}(v)=\varnothing$ при $u \neq v$, то $\nu_{\alpha}(u) \cap \nu_{\alpha}(v)=0$ при $u \neq v$, а так как $\bigcup_{z \in Z_{p}} \chi_{\theta(\alpha)}^{-1}(z)=G$, то $\bigvee_{z \in Z_{p}} \nu_{\alpha}(z)=1$, так что $\nu_{\alpha} \in Z_{p}[L]$.

Если $\varphi(\alpha)=\varphi(\beta)$, т.е. $\nu_{\alpha}=\nu_{\beta}$, то получаем, что $\langle\alpha\rangle=\langle\beta\rangle$ и $\theta(\alpha)=\theta(\beta)$. Согласно аксиоме 2) $\alpha=\beta$. Таким образом, $\varphi$ инъективно.

Необходимо доказать, что $\varphi$ - гомоморфизм, т.е. $\nu_{\alpha \beta}=\nu_{\alpha} \nu_{\beta}$ для любых $\alpha, \beta \in A$.

Если $(\alpha, \beta) \in \theta$, т.е. $\theta(\alpha)=\theta(\beta)$, то $\langle\alpha\rangle,\langle\beta\rangle \in S(\alpha)$ и $I(\alpha) \wedge I(\beta)=I(\alpha \beta)$ в решетке $L(\alpha)$ (см. лемму 7). В частности, $I(\alpha) \wedge I(\alpha)=I(\alpha)=I(\alpha \alpha)$. Используя лемму 1 , получаем

$$
\begin{aligned}
\left(\nu_{\alpha} \nu_{\beta}\right)(z) & =\bigvee_{z=u v}\left(\nu_{\alpha}(u) \wedge \nu_{\beta}(v)\right)=\bigvee_{z=u v}\left(\left(I(\alpha), \chi_{\theta(\alpha)}^{-1}(u)\right) \wedge\left(I(\beta), \chi_{\theta(\alpha}^{-1}(v)\right)\right) \\
& =\left(I(\alpha), \chi_{\theta(\alpha)}^{-1}(\sqrt{z})\right) \wedge\left(I(\beta), \chi_{\theta(\alpha)}^{-1}(\sqrt{z})\right)=\left(I(\alpha) \wedge I(\beta), \chi_{\theta(\alpha)}^{-1}(\sqrt{z})\right) \\
& =\left(I(\alpha \beta), \chi_{\theta(\alpha)}^{-1}(\sqrt{z})\right)=\left(I(\alpha \beta), \chi_{\theta(\alpha) \theta(\alpha)}^{-1}(z)\right) \\
& =\left(I(\alpha \beta), \chi_{\theta(\alpha) \theta(\beta)}^{-1}(z)\right)=\left(I(\alpha \beta), \chi_{\theta(\alpha \beta)}^{-1}(z)\right)=\left(\nu_{\alpha \beta}\right)(z)
\end{aligned}
$$

т.е. $\nu_{\alpha \beta}=\nu_{\alpha} \nu_{\beta}$ при $(\alpha, \beta) \in \theta$.

В случае $(\alpha, \beta) \notin \theta$ имеем

$$
\left(\nu_{\alpha} \nu_{\beta}\right)(z)=\bigvee_{z=u v}\left(\left(I(\alpha), \chi_{\theta(\alpha)}^{-1}(u)\right) \wedge\left(I(\beta), \chi_{\theta(\beta)}^{-1}(v)\right)\right)
$$

При построении вложения грушшы $G$ в булеву степень $Z_{p}\left[L^{*}\right]$ было показано, что

$$
\bigcup_{z=u v}\left(\chi_{\theta(\alpha)}^{-1}(u) \cap \chi_{\theta(\beta)}^{-1}(v)\right)=\chi_{\theta(\alpha \beta)}^{-1}(z)
$$

Значит, $\left(\nu_{\alpha} \nu_{\beta}\right)(z)$ является в $L$ элементом подрешетки $L(\alpha \beta, z)$. Это наименьший из ее элементов, в которых содержатся все элементы $\left(I(\alpha) \wedge I(\beta), \chi_{\theta(\alpha)}^{-1}(u) \cap \chi_{\theta(\beta)}^{-1}(v)\right)$, где $u v=z$. Пусть $\chi_{\theta(\alpha)}^{-1}(u) \cap \chi_{\theta(\beta)}^{-1}(v)=l_{u, v}$ в $L^{*}$. В каждой подрешетке $L\left(l_{u, v}\right)$ наибольшим элементом, содержашимся в $I(\alpha)$, является идеал

$$
J_{1}=I(\{(\langle\gamma\rangle,\langle\delta\rangle) \in S \times S \mid\langle\gamma\rangle \in I(\alpha), \text { или }\langle\delta\rangle \in I(\alpha), \text { или }\langle\gamma \delta\rangle \in I(\alpha)\}),
$$

а наибольшим элементом, содержашимся в $I(\beta)$, является идеал

$$
J_{2}=I(\{(\langle\gamma\rangle,\langle\delta\rangle) \in S \times S \mid\langle\gamma\rangle \in I(\beta), \text { или }\langle\delta\rangle \in I(\beta), \text { или }\langle\gamma \delta\rangle \in I(\beta)\}) .
$$


Нужно показать, что в подрешетке $L(\alpha \beta, z)$ наименьшим мажорантом для $J_{1} \cap J_{2}$ является $I(\alpha \beta)$.

Пусть $(\langle\gamma\rangle,\langle\delta\rangle) \in J_{1} \cap J_{2}$. Из возможных девяти случаев типичньпи являются следующие три:

a) $\langle\gamma\rangle \in I(\alpha),\langle\gamma\rangle \in I(\beta)$

б) $\langle\gamma\rangle \in I(\alpha),\langle\delta\rangle \in I(\beta)$;

в) $\langle\gamma\rangle \in I(\alpha),\langle\gamma \delta\rangle \in I(\beta)$.

В случае а) $\langle\gamma\rangle \leqslant\langle\alpha\rangle$ и $\langle\gamma\rangle \leqslant\langle\beta\rangle$, откуда, как установлено в лемме $6,\langle\gamma\rangle=\langle\gamma \gamma\rangle \leqslant\langle\alpha \beta\rangle$ и, значит, $(\langle\gamma\rangle,\langle\delta\rangle)<I(\alpha \beta)$. В случае б) $\langle\gamma \delta\rangle \leqslant\langle\alpha \beta\rangle$ и, значит, $(\langle\gamma\rangle,\langle\delta\rangle)<I(\alpha \beta)$. В случае в) согласно аксиоме 4$)$ получаем $\langle(\gamma \gamma) \delta\rangle \leqslant\langle\gamma(\gamma \delta)\rangle$, а так как $\langle\gamma(\gamma \delta)\rangle \leqslant\langle\alpha \beta\rangle$, то $\langle(\gamma \gamma) \delta\rangle \leqslant\langle\alpha \beta\rangle$ и, значит, $(\langle\gamma\rangle,\langle\delta\rangle)=(\langle\gamma \gamma\rangle,\langle\delta\rangle)<I(\alpha \beta)$.

Таким образом, элемент $I(\alpha \beta)$ подрешетки $L(\alpha \beta, z)$ является в $L$ верхней гранью для всех элементов $J_{1} \cap J_{2}$ из подрешеток $L\left(l_{u, v}\right)$, где $u v=z$. Учитьвая $(\langle\alpha\rangle,\langle\beta\rangle) \in J_{1} \cap J_{2}$, получаем, что $I(\alpha \beta)$ на самом деле будет наименьшей верхней гранью для этих элементов. Отсюда следует, что

$$
\left(\nu_{\alpha} \nu_{\beta}\right)(z)=\left(I(\alpha \beta), \chi_{\theta(\alpha \beta)}^{-1}(z)\right)=\nu_{\alpha \beta}(z)
$$

так что $\nu_{\alpha \beta}=\nu_{\alpha} \nu_{\beta}$ и при $(\alpha, \beta) \notin \theta$.

Это завершает доказательство теоремы 3 .

Поскольку всякая ассоциация с ассоциативным умножением является группой, пересечение класса групоидов, описанного в теореме 3 , с многообразием всех полугрупп совпадает с многообразием элементарных абелевых $p$-групп, $p>2$.

Естественный порядок в квазибулевых степенях сингулярных полугрупп, полурешеток и элементарных абелевых $p$-групा, $p>2$, может быть выражен через операцию умножения. Этот факт играет ключевую роль в построении соответствующих квазибулевых решеток. Многообразие элементарных 2-групп является в этом смысле исключением. Следующий пример показьвает, что в квазибулевых степенях элементарных 2-групп естественньй порядок не допускает интерпретации в терминах умножения.

ПримеР. Пусть $L^{*}=\{0,1, a, b, c, A, B, C\}$ - булева алгебра с атомами $a, b, c$ и коатомами $A=a^{\prime}, B=b^{\prime}, C=c^{\prime}$. Заменим элементы $b$ и $C$ двухэлементными множествами $\left\{b_{1}, b_{2}\right\}$ и $\left\{C_{1}, C_{2}\right\}$ соответственно. В полученном множестве определим следующие покрытия: элемент 1 непосредственно следует за каждьм из элементов $A, B, C_{2}$; каждьй из элементов $a, b_{1}, c$ является покрытием для 0; далее, $a<C_{1}, a<B, b_{1}<C_{1}, b_{1}<b_{2}$, $b_{2}<A, b_{2}<C_{2}, c<A, c<B, C_{1}<C_{2}$. Построенная диаграмма изображает квазибулеву решетку $L_{1}$, канонический $\wedge$-гомоморфизм которой на $L^{*}$ получается отождествлением элемента $b_{1}$ с элементом $b_{2}$ и элемента $C_{1}$ с элементом $C_{2}$. В квазибулевой степени $Z_{2}\left[L_{1}\right]$, где $Z_{2}=\{1,-1\}$, элементы $\mu_{1}=\left(C_{1}, c\right), \mu_{2}=\left(C_{2}, c\right), \nu_{1}=\left(B, b_{1}\right)$, $\nu_{2}=\left(B, b_{2}\right), \rho=(a, A), e=(1,0)$ образуют подгруппоид $G_{1}$ (отображение $\alpha: Z_{2} \rightarrow L$ мы записываем в виде вектора $(\alpha(1), \alpha(-1)))$. Таблица умножения в $G_{1}$, которое определяется формулой $(1)$, описывается равенствами

$$
\mu_{i} \mu_{j}=\nu_{i} \nu_{j}=\rho \rho=e, \quad \mu_{i} \nu_{j}=\rho, \quad \mu_{i} \rho=\nu_{i}, \quad \nu_{i} \rho=\mu_{i}
$$

$e$ - единица. При этом $\mu_{1}<\mu_{2}$ и $\nu_{1}<\nu_{2}$. Пусть $L_{2}$ есть решетка, диаграмма которой получается из диаграммы решетки $L_{1}$ взаимной заменой букв $C_{1}$ и $C_{2}, b_{1}$ и $b_{2}$ (т.е. в $L_{2}$ 
окажется $C_{2}<C_{1}, b_{2}<b_{1}$ и соответственно изменятся связанные с этими элементами покрытия). Тогда в $Z_{2}\left[L_{2}\right]$ элементы $\mu_{1}, \mu_{2}, \nu_{1}, \nu_{2}, \rho, e$, определенные выше, образуют подгруппоид $G_{2}$, таблица умножения которого совпадает с таблищей умножения группоида $G_{1}$, но в $G_{2}$ имеют место противоположные неравенства $\mu_{1}>\mu_{2}$ и $\nu_{1}>\nu_{2}$.

Вопрос о том, какие группиды с единицей допускают вложение в квазибулевы степени элементарных 2-групп, остается открытым (очевидньми необходимыми условиями являются коммутативность и вьполнимость тождества $x^{2}=e$ ).

\section{СПИСОК ЦИТИРОВАННОЙ ЛИТЕРАТУРЫ}

[1] Burris S. Boolean powers // Algebra Universalis. 1975. V. 5. № 3. P. 341-360.

[2] Пинус А.Г.Булевы конструкции в универсальной алгебре // УМН. 1992. Т. 41. № 4. C. $145-180$.

[3] Šešelja B. Lattice powers of unars // Зб. рад. прир. -матем. фак. Сер. матем. 1995. V. 25. № 2. P. 121-129.

[4] Салий В. Н. О квазибулевых степенях полугрупп // Упорядоченные множества и решетки. Вып. 10. Саратов, 1991. С. 104-108.

[5] Скорняков Л.А. (ред. ) Общая алгебра. Т. 2. М.: Наука, 1991.

[6] Салий В.Н. Квазибулевы степени сингулярных полугрупп // Изв. вузов. Матем. 1994. № 11. C. $67-74$.

[7] Salii V. N. On quasi-boolean powers of semilattices // Международная алгебраическая конференция памяти Д. К. Фаддеева. Тезисы докл. СПб., 1997. С. 112.

[8] Salii V. N. Quasi-boolean lattices and associations // Colloq. Math. Soc. János Bolyai. Lectures in Univ. Algebra. Szeged (Hungary), 1983. V. 43. Amsterdam: North-Holland, 1986. P. 429-454.

[9] Мальцев А.И. Об умножении классов алгебраических систем // Сиб. матем. ж. 1967. T. 8. №2. C. $346-365$.

[10] Salii V. N. Lattice extensions of algebras and Malcev products // Tatra Mt. Math. Publ. 1995. V. 5. P. 97-100.

[11] Фукс Л. Бесконечные абелевы группы. Т. 1. М.: Мир, 1974.

Саратовский государственный университет им. Н. Г. Черньшевского

Поступило 03.09 .98 